\title{
Boundary Behavior of the Bergman Kernel Function on Pseudoconvex Domains
}

\author{
By \\ Takeo OHSAWA*
}

\section{Hntroduction}

Let $D \subset \mathbb{C}^{n}$ be a bounded domain of holomorphy and let $H^{2}(D)$ be the set of square integrable holomorphic functions on $D$. The Bergman kernel function (cf. [1]) is defined by

where

$$
K_{D}(z):=\sup _{f \in H^{2}(D)-\{0\}}|f(z)|^{2} /\|f\|_{D}^{2}
$$

$\|f\|_{D}^{2}=\int_{D}|f(z)|^{2} d v \quad\left(d v\right.$ denotes the Lebesgue measure on $\left.\mathbb{C}^{n}\right)$.

$K_{D}(z)$ is regarded as a function measuring how large the space $H^{2}(D)$ can be. We are interested in the growth of $K_{D}$ near the boundary. Our motivation is the following theorem which has been proved by Hörmander and Diederich independently (see [2] and [4]).

Theorem 1 . If the boundary of $D$ is strictly pseudoconvex, then $K_{D}(z) \sim$ $d(z)^{-n-1}$. Here $d(z)=\inf _{x \in \partial D}|z-x|$ and $A \sim B$ means that both $A / B$ and $B / A$ are bounded.

From Theorem 1 and the definition of $K_{D}$ it can be easily seen that $K_{D}(z) \lesssim d(z)^{-n-1}$ if $\partial D$ is locally Lipschitz, where $A \gtrsim B$ means that $B / A$ is bounded. Further it has been shown by Pflug [6], [7] that if $D$ has a $C^{2}$ pseudoconvex boundary, then

$$
K_{D}(z) \gtrsim d(z)^{-2+\varepsilon}
$$

Here $\varepsilon$ is any positive number.

* Received February 6, 1982. Revised December 21, 1983.

Research Institute for Mathematical Sciences, Kyoto University, Kyoto 606, Japan. 
The present note may well be understood as a continuation of the above works. Let $\varphi$ be a defining function of a bounded domain $D$ with a $C^{2}$-pseudoconvex boundary in $\mathbb{C}^{n}$, let $x$ be a boundary point, and let

$$
\begin{aligned}
& N_{x}:=\left\{\left(\xi^{1}, \cdots, \xi^{n}\right) \in \mathbb{C}^{n} ; \sum_{i=1}^{n} \frac{\partial \varphi}{\partial z_{i}}(x) \xi^{i}=0, \sum_{i, j=1}^{n} \frac{\partial^{2} \varphi}{\partial z_{i} \partial z_{j}}(x) \xi^{j} \xi^{j}=0\right\}, \\
& \nu_{x}=\operatorname{dim}_{C} N_{x} .
\end{aligned}
$$

Under this situation we have the following theorem.

Main Theorem. Let $D$ be a bounded domain in $\mathbb{C}^{n}$ with $C^{4}$-pseudoconvex boundary. Fix $x \in \partial D$. Then, for any positive number $\varepsilon$,

$$
\inf _{z \in D} K_{D}(z)|z-x|^{n-v_{x}+1-z}>0 \text {. }
$$

Moreover if we set $S_{x}:=\left\{y ; \nu_{y}=\nu_{x}\right\}$ and

$$
m_{\varepsilon}^{x}(y):=\inf _{z \in D} K_{D}(z)|z-y|^{n-v_{x}+1-\varepsilon},
$$

then the function $y \mapsto m_{\varepsilon}^{x}(y)$ is continuous on $S_{x}$.

Corollary。 Let $\nu_{D}:=\sup _{x \in \partial D} \nu_{x^{\circ}} \quad$ Then, for any $\varepsilon>0$,

$$
K_{D}(z) \gtrsim d(z)^{-n+\nu_{D}-1+\varepsilon} \text { 。 }
$$

The main tool in the proof is a vanishing theorem on complete $\mathbb{K a ̈ h l e r}$ manifolds.

The author thanks to Professors K. Diederich and P. Pflug for their encouragement. Also many thanks to the referee for valuable criticisms.

\section{$\S$ 1. Localization lemma}

Let $D \subset \mathbb{C}^{n}$ be a bounded domain of holomorphy, let $x$ be a boundary point, and let $V$ and $U$ with $V \subset \subset U$ be two open neighbourhoods of $x$ in $\mathbb{C}^{n}$.

Localization lemma. There is a positive number $\delta$ such that for any point $y \in D \cap V$,

$$
\delta K_{D \cap U}(y) \leqq K_{D}(y) \leqq K_{D \cap U}(y) .
$$

Proof. Let $\chi: \mathbb{C}^{n} \rightarrow \mathbb{R}$ be a $\mathbb{C}^{\infty}$ function such that $\chi=1$ on a neighbourhood of $V$ and $\chi=0$ outside $U$. Let $z_{0} \in V \cap D$ be any point and let $f$ be a holomorphic function in $H^{2}(D \cap U)$ such that $\|f\|_{D \cap U}=1$ and $\left|f\left(z_{0}\right)\right|^{2}=K_{D \cap U}\left(z_{0}\right)$. We set $\alpha=\bar{\partial}(f \chi)$ on $U$ and $\alpha=0$ outside $U$. Then $\alpha$ is a $\bar{\partial}$-closed $(0,1)$-form 
defined on $D$ satisfying

$$
\left.\int_{D}\left|z-z_{0}\right|^{-2 n}\left|\exp \left(\left|z-z_{0}\right|^{2}\right)\right| \alpha\right|^{2} d v<C,
$$

where $C$ is a constant independent of $z_{0}$. Thus, by a well known theorem of Hörmander (cf. Theorem 2.2.1 in [4]), there is a function $\beta$ satisfying $\bar{\partial} \beta=\alpha$ and

$$
\int_{D}\left|z-z_{0}\right|^{-2 n} \exp \left(-\left|z-z_{0}\right|^{2}\right)|\beta|^{2} d v<C .
$$

Hence $\chi f-\beta$ is a holomorphic function defined on $D$ satisfying $\chi\left(z_{0}\right) f\left(z_{0}\right)-\beta\left(z_{0}\right)=f\left(z_{0}\right)$ and

$$
\int_{D}|\chi f-\beta|^{2} d v<2\left(C d^{2 n} \exp \left(d^{2}\right)+1\right),
$$

where $d$ denotes the diameter of $D$. Therefore if we set $\delta=\frac{1}{2}\left(C d^{2 n} \exp \left(d^{2}\right)+1\right)^{-1}$, we obtain (*).

\section{§2. Proof of Miain Theorem}

In what follows $D$ is a bounded domain in $\mathbb{C}^{n}$ with $\mathbb{C}^{4}$-pseudoconvex boundary and a defining function $\varphi$, and $x$ is a boundary point.

Proposition $\mathbb{1}$. Let $F$ be a holomorphic function on a neighbourhood of $\bar{D}$ such that $\{F=0\}$ is nonsingular, $\{F=0\} \cap \partial D=\{x\}$, and the zero-order of $\varphi \mid\{F=0\}$ is two for every tangent direction at $x$. Then, for every positive number $\varepsilon$,

$$
\int_{D}|F|^{-n-1+\varepsilon} d v<\infty
$$

Proof is easy.

We need the following proposition. (cf. [3]).

Proposition 2 (Diederich-Fornaess)。 There are positive numbers $L$ and $\eta_{0}$ such that for any positive number $\eta<\eta_{0}$, the function $-\left(d(z) \exp \left(-L|z|^{2}\right)\right)^{\eta}$ is strictly plurisubharmonic on $D \cap\left\{z_{0} ; d(z)\right.$ is $C^{2}$ at $\left.z_{0}\right\}$.

In what follows we take a coordinate $z=\left(z_{1}, \cdots, z_{n}\right)$ so that $x$ is the origin. We put $\nu=\nu_{x}, z^{\prime}=\left(z_{1}, \cdots, z_{\nu}, 0, \cdots, 0\right)$, and $z^{\prime \prime}=\left(0, \cdots, 0, z_{\nu+1}, \cdots, z_{n}\right)$. We may assume that the linear subspace $H=\left\{z ; z^{\prime}=0\right\}$ is transversal to $N_{x}$ and $\partial D$. 
We set $B(r)=\{z ;|z|<r\}$. We may assume that $B(1) \cap D \cap H$ is simply connected.

We put

$$
p(\varphi)=p(\varphi)\left(z^{\prime \prime}\right)=\sum_{i=\nu+1}^{n} \frac{\partial \varphi}{\partial z_{i}}(0) z_{i}+\sum_{i, j=v+1}^{n} \frac{\partial^{2} \varphi}{\partial z_{i} \partial z_{j}}(0) z_{i} z_{j} .
$$

Let $f_{\varepsilon}$ be a branch of $p(\varphi)^{(-n+y-1+\varepsilon) / 2}$ over $H \cap B(1) \cap D$, where $\varepsilon$ is a positive number satisfying $\varepsilon<2 /(n-\nu+1)$. Then, by Proposition 1 ,

$$
\int_{H \cap B(1) \cap D}\left|f_{\varepsilon}\right|^{2+\mathbb{\varepsilon}^{2}} d v<\infty
$$

We shall extend $\left.f_{\varepsilon}\right|_{H \cap B(1 / 2) \cap D}$ to a square integrable holomorphic function on $B(1 / 2) \cap D$. First, in virtue of Proposition 2, we may assume that for any sufficiently small $\eta,-(-\varphi)^{\eta}$ is strictly plurisubharmonic on $D$. If we set $\sigma\left(z_{1}, \cdots, z_{n}\right)=z^{\prime \prime}$, then we can find a positive number $C$ such that

$$
\sigma^{-1}(H \cap D) \supset\left\{z \in D ;-\varphi(z)>C\left|z^{\prime}\right|\right\} .
$$

Let $\chi$ be a $C^{\infty}$ function on $\mathbb{R}$ such that

$$
\chi(t)= \begin{cases}1 & \text { if } t \geqq 2 C \\ 0 & \text { if } t<C .\end{cases}
$$

We set $\lambda(z)=\chi\left(-\varphi(z) /\left|z^{\prime}\right|\right)$. Let $\Phi$ be a plurisubharmonic function on $B(1) \cap D$ defined by

$$
\Phi(z)=-\log \left(-\log \left|z^{\prime}\right|\right)-(-\varphi)^{\eta}+2 \nu \log \left|z^{\prime}\right|+|z|^{2} .
$$

Let $d v_{\Phi}$ and ||$_{\Phi}$ denote respectively the volume form and the length of forms with respect to $\partial \bar{\partial} \Phi$. We put

$$
\tilde{\alpha}= \begin{cases}\bar{\partial}\left(\lambda \sigma^{*} f_{\varepsilon}\right) \wedge d z_{1} \wedge \cdots \wedge d z_{n} \quad \text { on } & \sigma^{-1}(H \cap D \cap B(1)) \\ 0 & \text { otherwise, }\end{cases}
$$

and $\alpha=\left.\widetilde{\alpha}\right|_{D \cap B(1 / 2)}$. Then $\alpha$ is a $\bar{\partial}$-closed $(n, 1)$-form on $D \cap B(1 / 2)$.

Proposition 3. Let $\Phi$ and $\alpha$ be as above, then

$$
\int_{D \cap B(1 / 2)-H} e^{-\Phi}|\alpha|_{\Phi}^{2} d v_{\Phi}<\infty
$$

Proof. Choosing $\eta$ so small that $-(-\varphi)^{2 \eta}$ is strictly plurisubharmonic, we may assume that 


$$
\partial \bar{\partial}\left(-(-\varphi)^{\eta}\right) \geqq \eta^{2}(-\varphi)^{\eta-2} \partial \varphi \wedge \bar{\partial} \varphi
$$

Hence we have

$$
\begin{array}{r}
\partial \bar{\partial} \Phi \geqq \frac{\partial\left|z^{\prime}\right| \wedge \bar{\partial}\left|z^{\prime}\right|}{\left|z^{\prime}\right|^{2}\left(\log \left|z^{\prime}\right|\right)^{2}}+\eta^{2}(-\varphi)^{\eta-2} \partial \varphi \wedge \bar{\partial} \varphi+\sum_{i=1}^{n} d z_{i} \wedge d \bar{z}_{i} \\
\text { on } D \cap B(1)-H .
\end{array}
$$

Therefore,

$$
\begin{aligned}
& |\bar{\partial} \lambda(z)|_{\Phi}^{2}=\left|\chi^{\prime}\left(-\varphi(z) /\left|z^{\prime}\right|\right)\left(-\frac{\bar{\partial} \varphi}{\left|z^{\prime}\right|}+\frac{\varphi \bar{\partial}\left|z^{\prime}\right|}{\left|z^{\prime}\right|^{2}}\right)\right|_{\Phi}^{2} \\
\leqq & \frac{2 K^{2} C^{2}}{\eta^{2}}\left\{(-\varphi)^{-\eta}+\left(\log \left|z^{\prime}\right|\right)^{2}\right\} .
\end{aligned}
$$

Here, $K=\sup _{t \in R} \chi^{\prime}(t)$ and $\eta$ is chosen to be smaller than one. Since $\mid d z_{1} \wedge \ldots$ $\left.\wedge d z_{n}\right|_{\Phi} ^{2} d v_{\Phi}=\left|d z_{1} \wedge \cdots \wedge d z_{n}\right|^{2} d v$ we have

$$
|\alpha|_{\phi}^{2} d v_{\Phi} \leqq C_{1}(\eta)\left|f_{\varepsilon}(\sigma(z))\right|^{2}\left\{(-\varphi)^{-\eta}+\left(\log \left|z^{\prime}\right|\right)^{2}\right\} d v
$$

for some constant $C_{1}(\eta)$ depending on $\eta$. On the support of $\alpha$ we have $\left|z^{\prime}\right|<-\varphi(z) / C$. Hence

$$
|\alpha|_{\phi}^{2} d v_{\phi} \leqq C_{2}(\eta)\left|f_{\varepsilon}(\sigma(z))\right|^{2}(-\varphi)^{-\eta} d v
$$

for some constant $C_{2}(\eta)$. Therefore, for some constants $C_{3}(\eta), m$ and $M$, we have

$$
\begin{aligned}
& \int_{D \cap B(1 / 2)-H} e^{-\Phi}|\alpha|_{\Phi}^{2} d v_{\Phi} \\
\leqq & C_{3}(\eta) \int_{D \cap B(1) \cap H}\left|f_{\varepsilon}\right|^{2}\left|\varphi\left(z^{\prime \prime}\right)\right|^{-2 \eta}\left(\int_{B\left(M \varphi\left(z^{\prime \prime}\right)\right)-B\left(m \varphi\left(z^{\prime \prime}\right)\right)}\left|z^{\prime}\right|^{-2 \nu} d v_{1}\right) d v_{2} .
\end{aligned}
$$

Here $d v_{1}$ is the Lebesgue measure on $\left\{z^{\prime \prime}=\right.$ constant $\}$ and $d v_{2}$ is the Lebesgue measure on $H$. Hence,

$$
\begin{aligned}
& \int_{D \cap B(1 / 2)-H} e^{-\Phi}|\alpha|_{\Phi}^{2} d v_{\phi}
\end{aligned}
$$

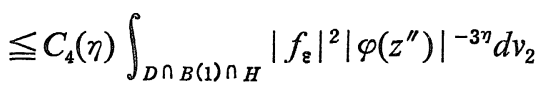

$$
\begin{aligned}
& \leqq C_{4}(\eta)\left(\int_{D \cap_{B}(1) \cap_{H}}\left|f_{\varepsilon}\right|^{2+\varepsilon^{2}} d v_{2}\right)^{2 /\left(2+\varepsilon^{2}\right)}\left(\int_{D \cap_{B}(1) \cap_{H}}\left|\varphi\left(z^{\prime \prime}\right)\right|^{-3\left(2+\varepsilon^{2}\right) \eta / \varepsilon^{2}} d v_{2}\right)^{\varepsilon^{2} /\left(2+\varepsilon^{2}\right)}
\end{aligned}
$$

for some constant $C_{4}(\eta)$. Hence, if $\eta$ is sufficiently small relative to $\varepsilon^{2}$, we have (**).

In [5] we have proved the following 
Proposition 4. Let $X$ be a complex manifold which admits a complete Kähler metric, and let $\Phi$ be a strictly plurisubharmonic function on $X$ of class $C^{4}$. Then, for any $\bar{\partial}$-closed $(n, 1)$-form $\alpha$ with $\int_{X} e^{-\Phi}|\alpha|_{\Phi}^{2} d v_{\Phi}<\infty$, we can find an $(n, 0)$ form $\beta$ satisfying $\bar{\partial} \beta=\alpha$ and $\int_{X} e^{-\Phi}|\beta|_{\Phi}^{2} d v_{\Phi} \leqq \int_{X} e^{-\Phi}|\alpha|_{\Phi}^{2} d v_{\Phi}$.

Since $D \cap B(1 / 2)-H$ admits a complete $\mathbb{K}$ ähler metric

$$
\sum_{i=1}^{n} d z_{i} \wedge d \bar{z}_{i}+\partial \bar{\partial}\left(-\log \left(-\log \left|z^{\prime}\right|\right)\right)+\partial \bar{\partial}(-1 / \varphi)+\partial \bar{\partial}\left(1 / 2-|z|^{2}\right)^{-1}
$$

Proposition 4 is applicable and we can find $\beta$ such that $\bar{\partial} \beta=\alpha$ and

$$
\int_{D \cap B(1 / 2)-H} e^{-\Phi}|\beta|_{\Phi}^{2} d v_{\Phi} \leqq \int_{D \cap B(1 / 2)-H} e^{-\Phi}|\alpha|_{\Phi}^{2} d v_{\Phi} .
$$

If we set

$$
f d z_{1} \wedge \cdots \wedge d z_{n}=\lambda \sigma^{*} f_{\varepsilon} d z_{1} \wedge \cdots \wedge d z_{n}-\beta
$$

we obtain a square integrable holomorphic function $f$ on $D \cap B(1 / 2)-H$ which naturally extends across $H$ and gives the desired extension of $f_{\mathrm{\varepsilon}}$.

Since the constructions of $f_{\varepsilon}$ and $f$ are uniform with respect to the choices of $x$ and $H$, we obtain the Main Theorem.

Q.E.D.

Question. Is it possible to drop $\varepsilon$ in Main Theorem?

\section{References}

[1] Bergman, S., The kernel function and the conformal mapping, Math. Surveys, No. 5 (1950).

[2] Diederich, K., Das Randverhalten der Bergmanschen Kernfunction und Metrik in streng pseudo-konvexen Gebieten, Math. Ann., 187 (1970), 9-36

[ 3 ] Diederich, K. and Fornaess, J.E., Pseudoconvex domains: Bounded strictly plurisubharmonic exhaustion functions, Invent. Math. 25 (1977), 129-141.

[4] Hörmander, L., $L^{2}$-estimates and existence theorems for the $\bar{\partial}$-operator. Acta Math. $1 \mathrm{H3}$ (1965), 89-152.

[5] Ohsawa, T., On complete Kähler domains with $C^{1}$-boundary, Publ. RIMS, Kyoto Univ. 16 (1980), 929-940.

[6] Pflug, P., Quadratintegrable holomorphe Funktionen und die Serre-Vermutung, Math. Ann. 216 (1975), 285-288.

[ 7 ] - Various applications of the existence of well growing holomorphic functions, Functional analysis, Holomorphy and approximation theory, J.A. Barroso (ed.), NorthHolland Publishing Company, 1982. 